\title{
Probing the structure of the Galactic disk through open clusters
}

\author{
Xiaoying Pang and Chenggang Shu
}

Key Laboratory for Astrophysics, Shanghai Normal University, Shanghai, P. R. China

\begin{abstract}
The WEBDA database of open clusters (hereafter OCs) in the Galaxy contains 970 OCs, of which 911 have age determinations, 920 have distance measurements, and 911 have colorexcess data. Base on the statistical analysis of global properties of open clusters, we investigate disk properties such as the height above the Galactic plane. We find that old open clusters (age $\geqslant 1$ Gyr) are preferentially located far from the Galactic plane with $\langle|z|\rangle \sim 394.5$ pc. They lie in the outer part of the Galactic disk. The young open clusters are distributed in the Galactic plane almost symmetrically with respect to the Sun, with a scale height perpendicular to the Galactic plane of 50.5 pc. The age distribution of open clusters can be fit approximately with a two-component exponential decay function: one component has an age scale factor of 225.2 Myr, and the other consists of longer-lived clusters with an age scale of $1.8 \mathrm{Gyr}$, which are smaller than those derived by Janes \& Phelps (1994) of 200 Myr and 4 Gyr for the young and old OCs, respectively. As a consequence of completeness effects, the observed radial distribution of OCs with respect to Galactocentric distance does not follow the expected exponential profile. Instead, it falls off both for regions external to the solar circle and more sharply towards the Galactic Center, which is probably due to giant molecular cloud disruption in the center. We simulate the effects of completeness, assuming that the observed distribution of the number of OCs with a given number of stars above the background is representative of the intrinsic distribution of OCs throughout the Galaxy. Two simulation models are considered, in which the intrinsic number of the observable stars are distributed (i) assuming the actual positions of the OCs in the sample, and (ii) random selection of OC positions. As a result, we derive completeness-corrected radial distributions which agree with an exponential disk throughout the observed Galactocentric distance in the range of $5-15 \mathrm{kpc}$, with scale lengths in the range of $1.6-2.8 \mathrm{kpc}$.
\end{abstract}

Keywords. Galaxy: disk, open clusters and associations: general

The full poster (in pdf format) is available at http://www. astro.iag.usp.br/〜iaus266/Posters/pPang.pdf. 